\title{
Hypernetworks: Multidimensional relationships in multilevel systems
}

\author{
J.H. Johnson \\ The Open University, Milton Keynes, MK17 6AA, UK
}

Received 28 March 2016 / Received in final form 26 July 2016

Published online 30 September 2016

\begin{abstract}
Networks provide a powerful way of modelling the dynamics of complex systems. Going beyond binary relations, embracing $n$-ary relations in network science can generalise many structures. This starts with hypergraphs and their Galois structures. Simplicial complexes generalise hypergraphs by adding orientation. Their multidimensional $q$-connectivity structure generalises connectivity in networks. Hypersimplices generalise simplices by making the relational structure explicit in the notation. This gives a new way of representing multilevel systems and their dynamics, leading to a new fragment-recombine operator to model the complex dynamics of interacting multilevel systems.
\end{abstract}

\section{Introduction}

Over the past decades networks have emerged as a powerful way of representing complex systems. It is suggested that network science will become even more powerful if it embraces $n$-ary relations between any number of vertices. This is very natural since it unifies well known structures in the network family as shown in Fig. 1. Less well known in the network community are simplicial families and hypernetworks, even though these are natural generalisations of networks and multiplex networks. It will be shown that hypersimplices and hypernetworks give a new way of representing multilevel objects and their dynamics. This allows the definition of new multilevel operations on multilevel systems with potentially rich mathematical properties and applications.

\section{Hypergraphs}

Rather remarkably, with some notable exceptions, the network community has barely engaged with $n$-ary relations between many vertices. For example in Fig. 2(a) the musical chord of $\mathrm{A}$ minor is made up of the notes $\{\mathrm{A}, \mathrm{C}, \mathrm{E}\}$, and all these notes are required. For example, removing $A$ from the chord of $A$ minor gives $\{C, E\}$. This is the same as removing the note $G$ from the chord of $C$ major, $\{C, E, G\}-\{G\}=\{C, E\}=$ $\{A, C, E\}-\{A\}$. Thus the notes needed to make the chord of $A$ minor are bound together by a 3-ary relation, and the chord is heard as a Gestalt, or whole. Play just the notes $\mathrm{C}$ and $\mathrm{E}$ and you get neither the chord of $\mathrm{A}$ minor nor the chord of $\mathrm{C}$ major. 


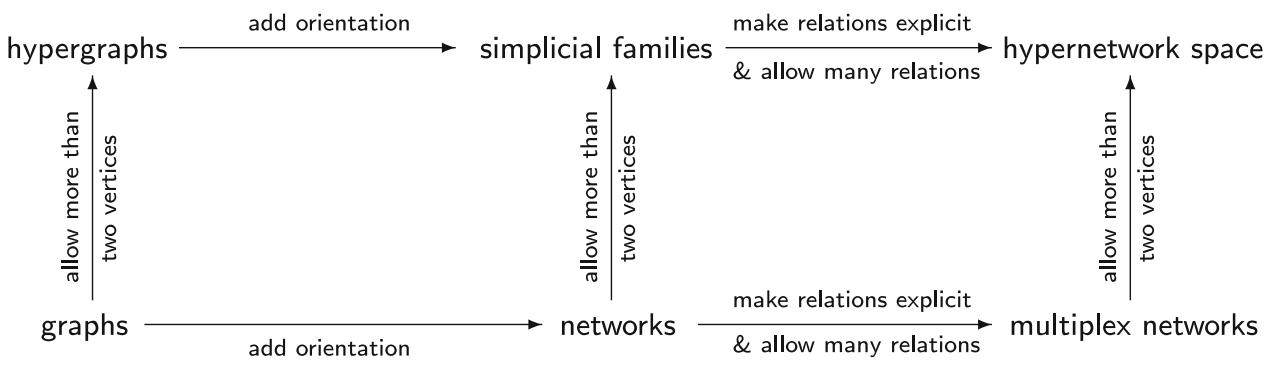

Fig. 1. The hypernetwork family of relational structures.

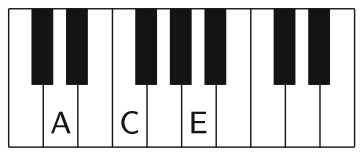

(a) the chord of A minor $\{A, C, E\}$

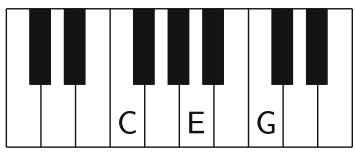

(b) the chord of $\mathrm{C}$ major $\{C, E, G\}$

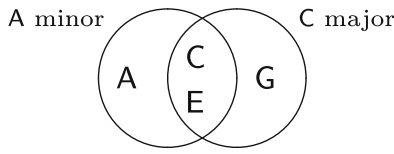

(c) the chords A minor and $C$ major as hypergraph edges

Fig. 2. 3-ary relations on musical notes make chords.

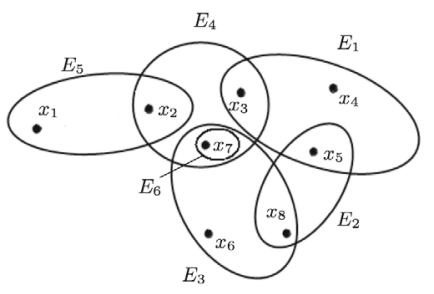

(a) $H_{E}(X, R)$

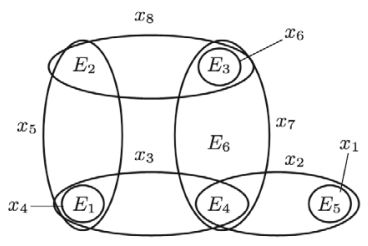

(b) $H_{X}(E, R)$

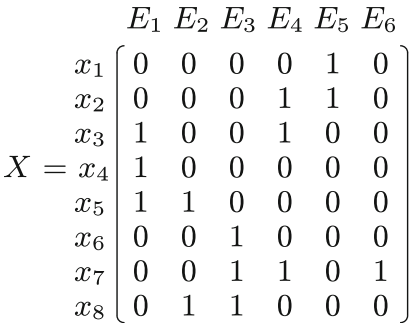

(c) the relation $R$

Fig. 3. The dual Berge hypergraphs.

Claude Berge gave the following definition [3,4]. Let $X=\left\{x_{1}, x_{2}, \ldots, x_{n}\right\}$ be a finite set. A hypergraph on $X$ is a family $H=\left(E_{1}, E_{2}, \ldots, E_{m}\right)$ of subsets of $X$ such that (1) $E_{i} \neq \emptyset$, and (2) $\bigcup_{i=1}^{m} E_{i}=X$. The elements $x_{1}, x_{2}, \ldots, x_{n}$ are called vertices, and the sets $E_{i}$ are the edges of the hypergraph.

Figure $3(\mathrm{c})$ shows a relation $R$ between vertices, $X$ and edges $E$ in Berge's book. Figure 3(a) shows the hypergraph here denoted as $H_{E}(X, R)$ which has edges $E_{j}$ as sets of vertices from $X$. Figure $3(\mathrm{~b})$ shows the dual hypergraph $H_{X}(E, R)$.

Hypergraphs give a way of modelling $n$-ary relations where $n$ can be any positive number. For example, Fig. 2(c) shows the chords $\mathrm{C}$ major and A minor as intersecting sets, or hypergraph edges. The generalisation of graphs to hypergraphs is shown on the left of Fig. 1.

\section{The Galois connection}

Let $R$ be a relation between $A$ and $B$. Let $R(a)=\{b \mid$ for all $b$ in $B$ with $a R b\}$ and $R(b)=\{a \mid$ for all $a$ in $A$ with $a R b\}$. Then $H_{A}(B, R) \stackrel{\text { def }}{=}\{R(a) \mid$ for all $a$ in $A\}$ and $H_{B}(A, R) \stackrel{\text { def }}{=}\{R(b) \mid$ for all $b$ in $B\}$ are called the dual hypergraphs of $R$. 


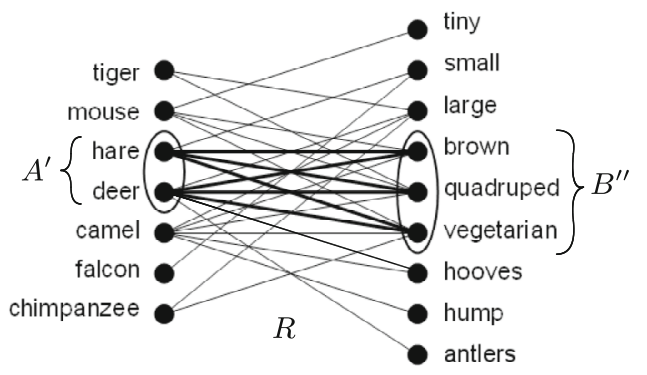

(a) $R(\{$ hare, deer $\})=R\left(A^{\prime}\right)=B^{\prime \prime}=$ \{brown, quadruped, vegetarian\}

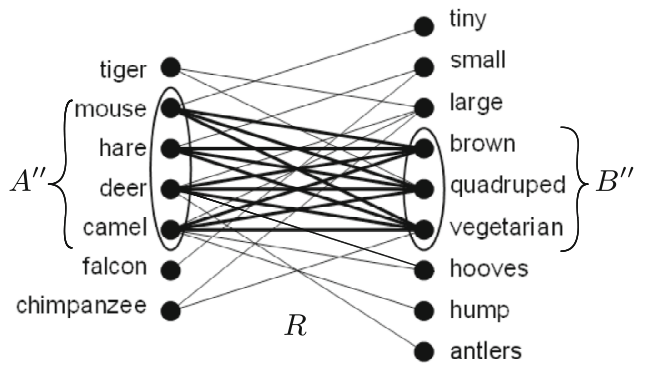

(b) $R\left(R(\{\right.$ hare, deer $\})=R\left(B^{\prime \prime}\right)=A^{\prime \prime}=$ \{mouse, hare, deer, camel $\}$

Fig. 4. Constructing the pair $\{$ mouse, hair, deer, camel $\} \leftrightarrow\{$ brown, quadruped, vegetarian $\}$.

Let $A^{\prime}$ be a subset of $A, A^{\prime} \subseteq A$ and let $B^{\prime}$ be a subset of $B, B^{\prime} \subseteq B$. Let

$$
\begin{aligned}
& R\left(A^{\prime}\right) \stackrel{\text { def }}{=}\left\{b \mid \text { for all } b \text { in } B \text { with } b \text { related to all } a \text { in } A^{\prime}\right\}=\bigcap_{a \in A^{\prime}} R(a) \\
& R\left(B^{\prime}\right) \stackrel{\text { def }}{=}\left\{a \mid \text { for all } a \text { in } A \text { with } a \text { related to all } b \text { in } B^{\prime}\right\}=\bigcap_{b \in B^{\prime}} R(b)
\end{aligned}
$$

For any $A^{\prime} \subseteq A$ let $B^{\prime \prime}=R\left(A^{\prime}\right)$. For $B^{\prime \prime} \neq \emptyset$ let $A^{\prime \prime}=R\left(B^{\prime \prime}\right)$. Then $\left(A^{\prime \prime}, B^{\prime \prime}\right)$ is defined to be a Galois pair of $R$, also written as $A^{\prime \prime} \leftrightarrow B^{\prime \prime}$. Let

$\mathcal{H}_{A}(B, R)=\left\{B^{\prime \prime} \mid\right.$ there exists a Galois pair $\left.A^{\prime \prime} \leftrightarrow B^{\prime \prime}\right\}$, and

$\mathcal{H}_{B}(A, R)=\left\{A^{\prime \prime} \mid\right.$ there exists a Galois pair $\left.A^{\prime \prime} \leftrightarrow B^{\prime \prime}\right\}$. Then:

\section{Proposition (Galois Connection)}

(i) $H_{A}(B, R) \subseteq \mathcal{H}_{A}(B, R)$ and $H_{B}(A, R) \subseteq \mathcal{H}_{B}(A, R)$, and

(ii) There is a one-to-one correspondence between $\mathcal{H}_{A}(B, R)$ and $\mathcal{H}_{B}(A, R)$.

$\mathcal{H}_{A}(B, R)$ and $\mathcal{H}_{B}(A, R)$ are defined to be the dual Galois hypergraphs of the relation $R$ between $A$ and $B$.

This is illustrated in Fig. 4 where $A$ is a set of animals and $B$ is a set of descriptive features. Let $A^{\prime}=\{$ hare, deer $\}$. Then as shown in Fig. $4(\mathrm{a}) R\left(A^{\prime}\right)=$ brown, quadruped, vegetarian $\}=B^{\prime \prime}$. The left of Fig. $4(\mathrm{~b})$ shows that $R\left(B^{\prime \prime}\right)=$ mouse, hare, deer, camel $\}=A^{\prime \prime}$. Thus, starting with $A^{\prime} \stackrel{\text { def }}{=}\{$ hare, deer $\}$ we have constructed the Galois pair

$$
A^{\prime \prime}=\{\text { mouse, hare, deer, camel }\} \leftrightarrow\{\text { brown, quadruped, vegetarian }\}=B^{\prime \prime} .
$$

By construction $A^{\prime \prime}$ is "maximal", the largest set related to all the $b$ in $B^{\prime \prime}$. $B^{\prime \prime}$ is also "maximal", since every $b$ related to every member of $A^{\prime \prime}$ is a member of $B^{\prime \prime}$. This follows from $A^{\prime \prime}$ being a superset of $A^{\prime}: A^{\prime \prime} \supseteq A^{\prime}$ implies $\cap_{a \in A^{\prime \prime}} R(a) \subseteq$ $\cap_{a \in A^{\prime}} R(a)=B^{\prime \prime}$.

Figure 5 illustrates the connection between the Galois pair just formed. On the left are hypergraph edges for the four animals forming a star with their three shared features as a $h u b$. On the right the three features form a star with the four animals as a hub. The hubs form the Galois pair.

Figure 6(a) shows Escher's picture Sky and Water in which the birds at the top of the picture seem to change into fish at the bottom. Figure 6(b) shows the various shapes that appear in the picture. 


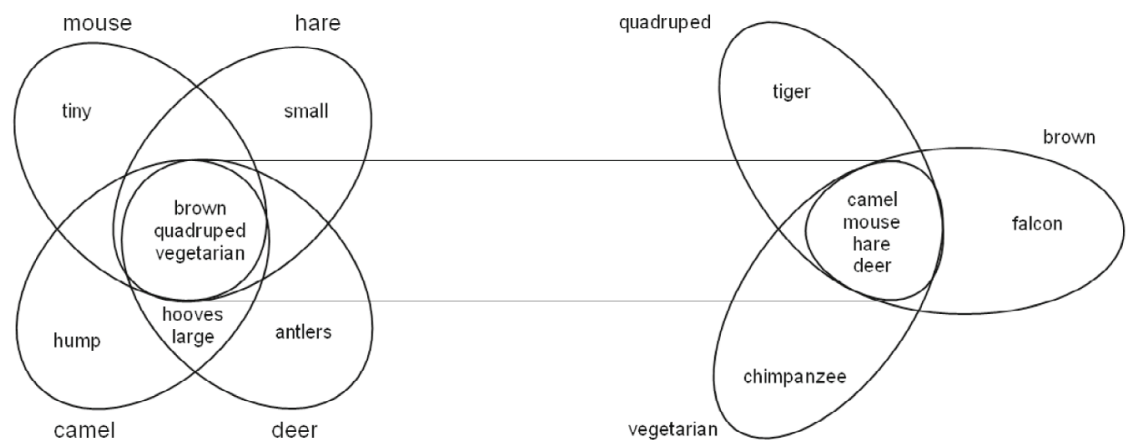

Fig. 5. The Galois pair as a star-hub configuration.

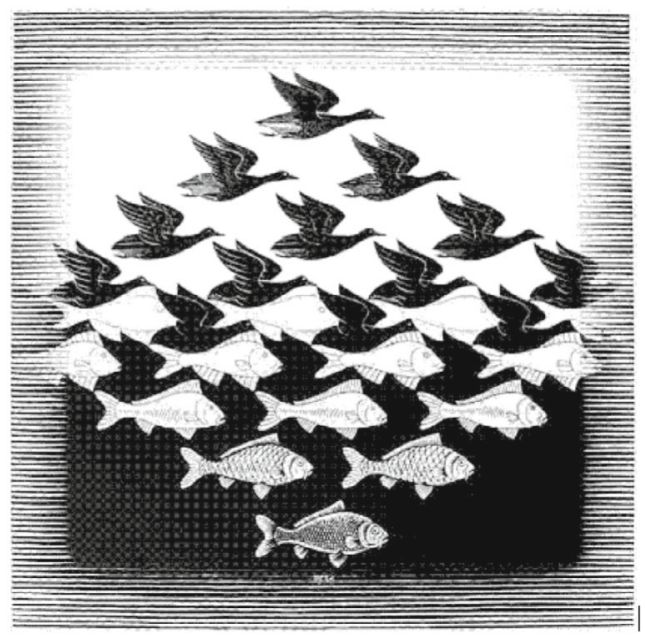

(a) Escher's Sky and Water

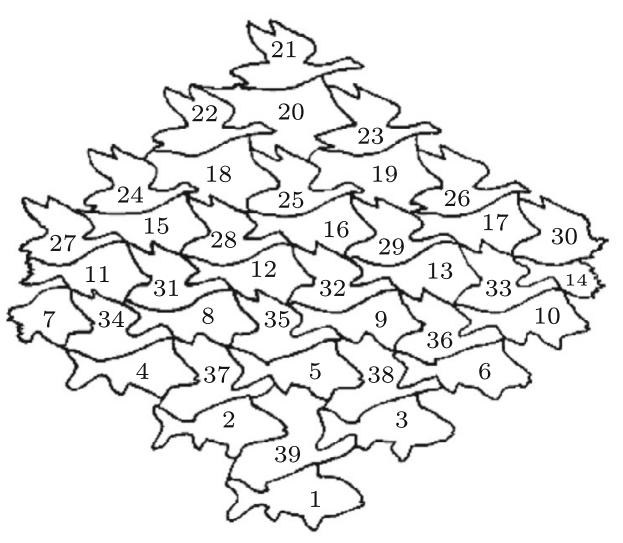

(b) fish and bird shapes

Fig. 6. The shapes and features abstracted from Escher's Sky and Water.

Table 1 below shows a relation between the set of thirty nine shapes and a set of twelve descriptors. The Galois pairs correspond to what are called maximal rectangles in the incidence matrix. Note that the rows of the incidence matrix can be swapped without losing information and the columns can be swapped without losing information. If $A^{\prime \prime}$ and $B^{\prime \prime}$ form a Galois pair the rows can be swapped so that all of those corresponding to $A^{\prime \prime}$ are contiguous. Similarly the columns can be swapped so that all the columns corresponding to $B^{\prime \prime}$ are contiguous. When this is done the entries corresponding to $A^{\prime \prime}$ and $B^{\prime \prime}$ in the matrix form a block of ones. These blocks are called maximal rectangles because rearranging other rows and columns cannot make them bigger.

In Table 1 the features and shapes have been arranged to show some of the larger maximal rectangles corresponding to star-hub Galois pairs, including:

$\langle 1,2,3,4,5,6\rangle \longleftrightarrow \quad\langle$ scales, mouth, gills, fish-tails, fins, fish-shape, eye $\rangle$

$\langle 1,2,3,4,5,6,8,9,10,11,12,13\rangle \quad\langle\quad$ fish-tails, fins, fish-shape, eye

$\langle 21,22,23,24,25,26,28,29\rangle \quad \longleftrightarrow \quad\langle$ eye, duck-shape, two-wings, feathers, beak, legs $\rangle$

$\langle 21,22,23,24,25,26,28,29,27,31,32,33\rangle \quad \longleftrightarrow \quad\langle$ eye, duck-shape, two-wings $\rangle$ 
Table 1. The incidence matrix for the animal - feature relation.

\begin{tabular}{l|lllllllllllllllllllllllllllllllllllllllllllllllllllllllllllllllllllll} 
Shapes: & 1 & 2 & 3 & 4 & 5 & 6 & 8 & 9 & 10 & 11 & 12 & 13 & 7 & 21 & 22 & 23 & 24 & 25 & 26 & 28 & 29 & 27 & 31 & 32 & 33 & 30 & 34 & 35 & 36 & 37 & 38 & 14 & 15 & 16 & 17 & 18 & 19 & 20 & 39 \\
\hline scales & 1 & 1 & 1 & 1 & 1 & 1 & 0 & 0 & 0 & 0 & 0 & 0 & 0 & 0 & 0 & 0 & 0 & 0 & 0 & 0 & 0 & 0 & 0 & 0 & 0 & 0 & 0 & 0 & 0 & 0 & 0 & 0 & 0 & 0 & 0 & 0 & 0 & 0 & 0 \\
mouth & 1 & 1 & 1 & 1 & 1 & 1 & 1 & 1 & 1 & 0 & 0 & 0 & 1 & 0 & 0 & 0 & 0 & 0 & 0 & 0 & 0 & 0 & 0 & 0 & 0 & 0 & 0 & 0 & 0 & 0 & 0 & 0 & 0 & 0 & 0 & 0 & 0 & 0 & 0 \\
gills & 1 & 1 & 1 & 1 & 1 & 1 & 1 & 1 & 1 & 0 & 0 & 0 & 1 & 0 & 0 & 0 & 0 & 0 & 0 & 0 & 0 & 0 & 0 & 0 & 0 & 0 & 0 & 0 & 0 & 0 & 0 & 0 & 0 & 0 & 0 & 0 & 0 & 0 & 0 \\
fish-tail & 1 & 1 & 1 & 1 & 1 & 1 & 1 & 1 & 1 & 1 & 1 & 1 & 0 & 0 & 0 & 0 & 0 & 0 & 0 & 0 & 0 & 0 & 0 & 0 & 0 & 0 & 0 & 0 & 0 & 0 & 0 & 1 & 0 & 0 & 0 & 0 & 0 & 0 & 0 \\
fins & 1 & 1 & 1 & 1 & 1 & 1 & 1 & 1 & 1 & 1 & 1 & 1 & 1 & 0 & 0 & 0 & 0 & 0 & 0 & 0 & 0 & 0 & 0 & 0 & 0 & 0 & 0 & 0 & 0 & 0 & 0 & 0 & 0 & 0 & 0 & 0 & 0 & 0 & 0 \\
fish-shape & 1 & 1 & 1 & 1 & 1 & 1 & 1 & 1 & 1 & 1 & 1 & 1 & 0 & 0 & 0 & 0 & 0 & 0 & 0 & 0 & 0 & 0 & 0 & 0 & 0 & 0 & 0 & 0 & 0 & 0 & 0 & 0 & 1 & 1 & 1 & 1 & 1 & 0 & 0 \\
eye & 1 & 1 & 1 & 1 & 1 & 1 & 1 & 1 & 1 & 1 & 1 & 1 & 1 & 1 & 1 & 1 & 1 & 1 & 1 & 1 & 1 & 1 & 1 & 1 & 1 & 0 & 0 & 0 & 0 & 0 & 0 & 0 & 0 & 0 & 0 & 0 & 0 & 0 & 0 \\
duck-shape & 0 & 0 & 0 & 0 & 0 & 0 & 0 & 0 & 0 & 0 & 0 & 0 & 0 & 1 & 1 & 1 & 1 & 1 & 1 & 1 & 1 & 1 & 1 & 1 & 1 & 0 & 1 & 1 & 1 & 1 & 1 & 0 & 0 & 0 & 0 & 0 & 0 & 0 & 0 \\
two-wings & 0 & 0 & 0 & 0 & 0 & 0 & 0 & 0 & 0 & 0 & 0 & 0 & 0 & 1 & 1 & 1 & 1 & 1 & 1 & 1 & 1 & 1 & 1 & 1 & 1 & 1 & 0 & 0 & 0 & 0 & 0 & 0 & 0 & 0 & 0 & 0 & 0 & 0 & 0 \\
feathers & 0 & 0 & 0 & 0 & 0 & 0 & 0 & 0 & 0 & 0 & 0 & 0 & 0 & 1 & 1 & 1 & 1 & 1 & 1 & 1 & 1 & 1 & 0 & 0 & 0 & 0 & 0 & 0 & 0 & 0 & 0 & 0 & 0 & 0 & 0 & 0 & 0 & 0 & 0 \\
beak & 0 & 0 & 0 & 0 & 0 & 0 & 0 & 0 & 0 & 0 & 0 & 0 & 0 & 1 & 1 & 1 & 1 & 1 & 1 & 1 & 1 & 1 & 0 & 0 & 0 & 0 & 0 & 0 & 0 & 0 & 0 & 0 & 0 & 0 & 0 & 0 & 0 & 0 & 0 \\
legs & 0 & 0 & 0 & 0 & 0 & 0 & 0 & 0 & 0 & 0 & 0 & 0 & 0 & 1 & 1 & 1 & 1 & 1 & 1 & 1 & 1 & 0 & 0 & 0 & 0 & 0 & 0 & 0 & 0 & 0 & 0 & 0 & 0 & 0 & 0 & 0 & 0 & 0 & 0
\end{tabular}
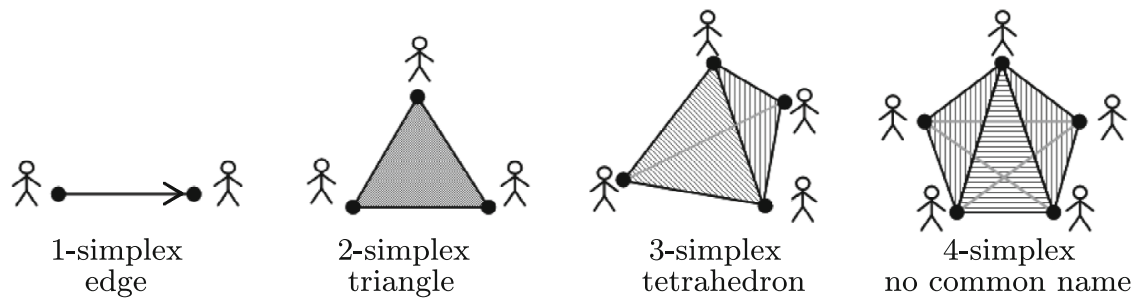

Fig. 7. Simplices generalise network edges to multidimensional space.

There are other Galois pairs whose rectangles are not outlined in Table 1, e.g.

$\langle 1,2,3,4,5,6,8,9,10\rangle \longleftrightarrow \quad\langle$ mouth, gills, fish-tails, fins, fish-shape, eye $\rangle$

$\langle 21,22,23,24,25,26,28,29,27\rangle \longleftrightarrow\langle$ eye, duck-shape, two-wings, feathers, beak $\rangle$

$\langle 1,2,3,4,5,6,8,9,10,7\rangle \longleftrightarrow\langle$ mouth, gills, fins, eye $\rangle$

Galois pairs play an important role in the move from binary to $n$-ary relations $[8,9]$.

\section{Simplicial families and complexes}

Hypergraphs provide an excellent way of representing $n$-ary relations. Their settheoretic nature makes it easy to formulate structures such as the Galois pairs. However there is the problem. For example, when $R$ is a relation such as "likes" on a set of people, since $\{a, b\}=\{b, a\}$ it follows that if $a$ likes $b$ then $b$ likes $a$. In general this is not true and it is necessary for edges to be oriented to reflect asymmetry. This is achieved in networks which allow $\langle a, b\rangle \neq\langle b, a\rangle$.

In algebraic topology, given a set of vertices $V$, an abstract p-simplex is an ordered subset of those vertices $\left\langle v_{0}, v_{1}, \ldots, v_{p}\right\rangle$. A $p$-simplex has an associated geometric realisation as a $p$-dimensional polyhedron, as shown in Fig. 7. A set of simplices is called a simplicial family.

The orientation of simplices can be very important and in general $\left\langle\ldots, v_{i}, \ldots, v_{j}, \ldots\right\rangle \neq\left\langle\ldots, v_{i}, \ldots, v_{j}, \ldots\right\rangle$. This is what distinguishes simplices from hypergraph edges. A hypergraph can be made into a simplicial family as follows. Let $V$ be the vertex set of a hypergraph. Let the members of $V$ have an arbitrary but fixed 


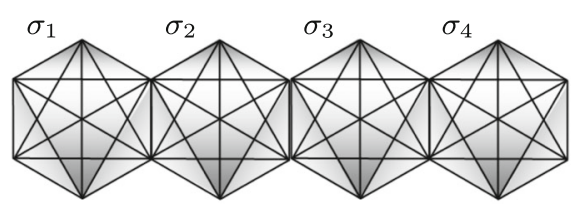

(a) 1-connected 5-dimensional simplices

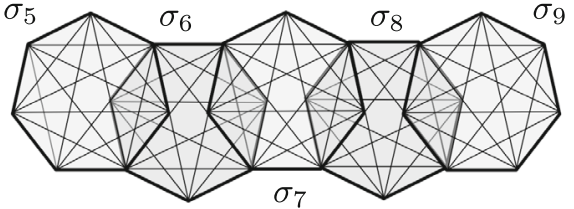

(b) 3-connected 6-dimensional simplices

Fig. 8. $q$-connected simplices.

numbering, $v_{(1)}, v_{(2)}, v_{(3)}$, etc. Call this the natural ordering. Let the hypergraph edge $\left\{\ldots, v_{i}, \ldots, v_{j}, \ldots\right\}$ have the natural ordering if for all $i$ and $j$, there exists $v_{(\alpha)}=v_{i}$ and $v_{(\beta)}=v_{j}$ such that $\alpha<\beta$ for $i<j$. In this way every hypergraph can be considered to be a simplicial family.

A $q$-simplex $\sigma=\left\langle v_{0}, v_{1}, \ldots, v_{q}\right\rangle$ is a $q$-dimensional face of $\sigma^{\prime}=\left\langle v_{0}^{\prime}, v_{1}^{\prime}, \ldots, v_{p}^{\prime}\right\rangle$ if $\left\{v_{0}, v_{1}, \ldots, v_{q}\right\} \subseteq\left\{v_{0}^{\prime}, v_{1}^{\prime}, \ldots, v_{p}^{\prime}\right\}$, e.g. a tetrahedron has four 2-dimensional faces (triangles), six 1-dimensional faces (edges) and four 0-dimensional faces (vertices).

A set of simplices with all its faces is a simplicial complex. Simplicial complexes are used extensively in algebraic topology but this important aspect of hypernetworks will not be developed here. For more details see (Johnson, 2014 [9]).

Two simplices are said to be $q$-near when they share a $q$-dimensional face. Two simplices are said to be $q$-connected if there is a chain of pairwise $q$-near simplices between them. For example, in Fig. 8(a) the 5-dimensional simplices $\sigma_{1}$ and $\sigma_{2}$ are 1-near, and $\sigma_{1}$ and $\sigma_{4}$ are 1-connected through $\sigma_{2}$ and $\sigma_{3}$. In Fig. 8(b) the 6dimensional simplices $\sigma_{5}$ and $\sigma_{6}$ share a tetrahedral face and are 3-near. $\sigma_{5}$ and $\sigma_{9}$ are 3 -connected through $\sigma_{6}, \sigma_{7}$ and $\sigma_{8}$, even though they share no vertices.

\section{Q-analysis}

Let $F$ be a simplicial family. The dimension of the family is the highest dimension of all its simplices. Being $q$-connected is an equivalence relation on the set of $p$-simplices of $F, p \geq q$, and partitions them into sets of $q$-connected components. A listing of those $q$-connected components for each $q$ from the dimension of $F$ to zero is called a Q-analysis, e.g. for Escher's Sky and Water animal-feature relation the Q-analysis is:

$$
\begin{aligned}
& q=\mathbf{6}\{1,2,3,4,5,6\} \\
& q=\mathbf{5}\{1,2,3,4,5,6,8,9,10\} \quad\{21,22,23,24,25,26,28,29\} \\
& q=\mathbf{4}\{1,2,3,4,5,6,8,9,10\} \quad\{21,22,23,24,25,26,28,29,27\} \\
& q=\mathbf{3}\{1,2,3,4,5,6,8,9,10,11,12,13,7\} \quad\{21,22,23,24,25,26,28,29,27\} \\
& q=\mathbf{2}\{1,2,3,4,5,6,8,9,10,11,12,13,7\} \quad\{21,22,23,24,25,26,28,29,27,31,32,33\} \\
& q=\mathbf{1}\{1,2,3,4,5,6,8,9,10,11,12,13,7\} \quad\{21,22,23,24,25,26,28,29,27,31,32,33\} \\
& q=\mathbf{0}\{1,2,3,4,5,6,8,9,10,11,12,13,7,14,15,16,17,18,19,21,22,23,24,25,26,28, \\
&29,27,31,32,33,34,35,36,37,38\}
\end{aligned}
$$

Figure 9 shows a graphical way of displaying the $Q$-analysis using skyscraper diagrams. The top diagram shows that the bird and fish shapes form distinct components. Removing the "eye" descriptor creates two disconnected subfamilies, one with fish shapes and the other with duck shapes. Thus the transition from ducks at the top of Escher's picture to the fish at the bottom does not involve morphing from one shape to the other. Instead the picture is tiled by shapes, half of which get more duck-like towards the top and half of which get more fish-like towards the bottom. 


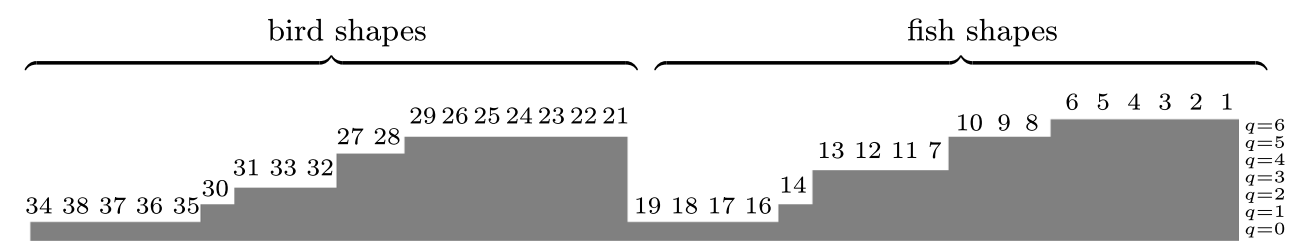

(a) Skyscraper diagram for the Q-analysis of $F_{\text {Shapes }}($ Descriptors).

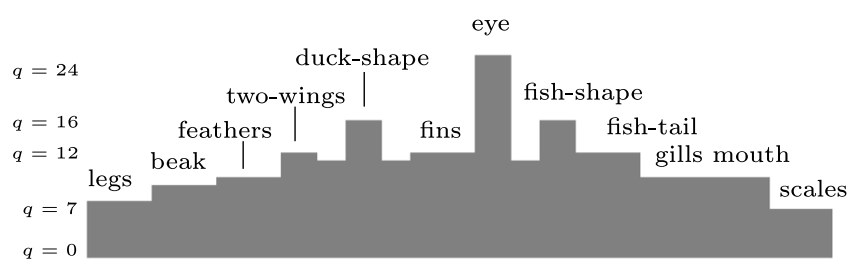

(b) Skyscraper diagram for the Q-analysis of $F_{\text {Descriptors }}($ Scales).

Fig. 9. The skyscraper diagrams for the shapes - features Q-analyses.

Table 2. The most popular answers selected by the 45 students.

$\begin{array}{llllllll}\begin{array}{l}\text { majority } \\ \text { answer }\end{array} & \begin{array}{l}\text { number of } \\ \text { students }\end{array} & \begin{array}{l}\text { majority } \\ \text { answer }\end{array} & \begin{array}{l}\text { number of } \\ \text { students }\end{array} & \begin{array}{l}\text { majority } \\ \text { answer }\end{array} & \begin{array}{l}\text { number of } \\ \text { students }\end{array} & \begin{array}{l}\text { majority } \\ \text { answer }\end{array} & \begin{array}{l}\text { number of } \\ \text { students }\end{array} \\ q_{1}-\mathrm{C}_{1} & 4396 \% & q_{6}-\mathrm{F}_{6} & 2453 \% & q_{11}-\mathrm{F}_{11} & 3782 \% & q_{16}-\mathrm{D}_{16} & 3169 \% \\ q_{2}-\mathrm{B}_{2} & 3271 \% & q_{7}-\mathrm{C}_{7} & 4089 \% & q_{12}-\mathrm{C}_{12} & 4191 \% & q_{17}-\mathrm{G}_{17} & 4293 \% \\ q_{3}-\mathrm{A}_{3} & 45100 \% & q_{8}-\mathrm{D}_{8} & 2658 \% & q_{13}-\mathrm{B}_{13} & 3578 \% & q_{18}-\mathrm{D}_{18} & 3373 \% \\ q_{4}-\mathrm{G}_{4} & 3476 \% & q_{9}-\mathrm{E}_{9} & 3680 \% & q_{14}-\mathrm{D}_{14} & 3067 \% & q_{19}-\mathrm{C}_{19} & 3067 \% \\ q_{5}-\mathrm{C}_{5} & 45100 \% & q_{10}-\mathrm{A}_{10} & 3476 \% & q_{15}-\mathrm{F}_{15} & 2658 \% & q_{20}-\mathrm{F}_{20} & 3680 \%\end{array}$

\section{Example: Detecting hidden information by connectivity}

In a multiple choice test, 45 students answered 20 questions. For each question, $q_{i}$ they selected 1 from 7 answers, $A_{i}, B_{i}, C_{i}, D_{i}, E_{i}, F_{i}, G_{i}$. Usually in multiple choice tests the majority of students get the correct answers. The most popular answers are shown in Table 2. This shows that for all but three of the questions, more than $66 \%$ of the students chose the same answer. For questions $q_{6}, q_{8}$ and $q_{15}$ the answers selected for were $\mathrm{F}_{6}(53 \%), \mathrm{D}_{8}(58 \%)$ and $\mathrm{F}_{15}(58 \%)$ respectively. Are these answers correct?

Let $\sigma\left(s_{i}\right)$ be the simplex with the answers of student $s_{i}$ as vertices, and $F_{S}(Q ; R)$ be the family $\left\{\sigma\left(s_{i}\right) \mid i=1, \ldots, 45\right\}$. Its $Q$-analysis is given as the skyscraper diagram in Fig. 10. This shows that six students $s_{42}, s_{16}, s_{43}, s_{4}, s_{32}, s_{19}$ all gave exactly the same answers and had simplex:

$\sigma_{*} \stackrel{\text { def }}{=}\left\langle\mathrm{C}_{1}, \mathrm{~B}_{2}, \mathrm{~A}_{3}, \mathrm{G}_{4}, \mathrm{C}_{5}, \mathrm{E}_{6}, \mathrm{C}_{7}, \mathrm{D}_{8}, \mathrm{E}_{9}, \mathrm{~A}_{10}, \mathrm{~F}_{11}, \mathrm{C}_{12}, \mathrm{~B}_{13}, \mathrm{D}_{14}, \mathrm{~F}_{15}, \mathrm{D}_{16}, \mathrm{G}_{17}, \mathrm{D}_{18}, \mathrm{C}_{19}, \mathrm{~F}_{20}\right\rangle$.

Generally good students will be highly connected through correct answers, while poor students will be relatively disconnected because their incorrect answers are scattered between six distractors. This suggests the vertices of $\sigma_{*}$ are all correct answers.

$\left\langle\mathrm{D}_{8}\right\rangle$ and $\left\langle\mathrm{F}_{15}\right\rangle$ are both vertices of $\sigma_{*}$ suggesting that they are correct answers, despite only being selected by $58 \%$ of the students. However, the $53 \%$ majority answer $\left\langle\mathrm{F}_{6}\right\rangle$ is not a vertex of $\sigma_{*}$, with $\left\langle\mathrm{E}_{6}\right\rangle$ selected instead by these six students. There are no other 19-connected components (students sharing 20 answer vertices as a 19-dimensional simplex) which suggests the minority answer $\left\langle\mathrm{E}_{6}\right\rangle$ is correct.

At the left of Fig. 10 the students, $s_{12}, s_{26}, s_{38}$ and $s_{41}$ are the most disconnected and likely to be the weakest and make the most errors. They all gave the majority 
students

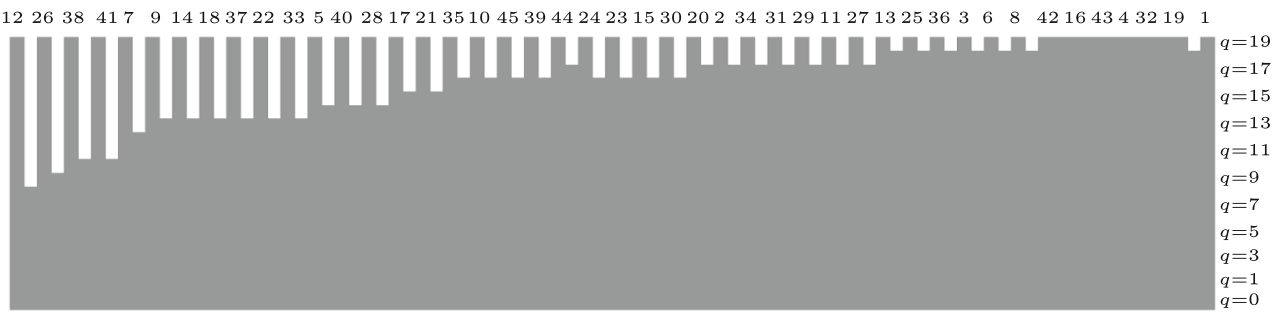

Fig. 10. Q-analysis of the student-questions relation, $F_{S}(Q ; R)$ showing connected students.

answer. Of the 14 students on the left of Fig. $10, s_{12}$ to $s_{28}$, twelve $(86 \%)$ gave the majority answer $\left\langle\mathrm{F}_{6}\right\rangle$. This is further strong evidence that this answer is incorrect.

Looking back to the top right of Fig. 10, of the twenty most highly connected students $s_{20}$ to $s_{1}$, sixteen $(75 \%)$ gave the minority answer, suggesting it is correct.

Thus the $Q$-analysis gives very strong evidence that the majority answer $\left\langle\mathrm{F}_{6}\right\rangle$ is incorrect and that the minority answer $\left\langle\mathrm{E}_{6}\right\rangle$ is correct.

Having reached this conclusion solely on the basis of the $Q$-analysis, it can be tested by looking at Question 6: "A body moves in such a way that its speed (in miles per hour) after $t$ hours is $4 t^{3}$. How far has it travelled after 3 hours?" It gives the options $\left(\mathrm{A}_{6}\right) 16$ miles, $\left(\mathrm{B}_{6}\right) 27$ miles, $\left(\mathrm{C}_{6}\right) 54$ miles, $\left(\mathrm{D}_{6}\right) 64$ miles, $\left(\mathrm{E}_{6}\right) 81$ miles, $\left(\mathrm{F}_{6}\right) 108$ miles, and $\left(\mathrm{G}_{6}\right) 243$ miles. The stronger students correctly integrated $4 t^{3}$ and substitute 3 into $t^{4}$ to give 81 miles $\left(\mathrm{E}_{6}\right)$, while the weaker student incorrectly substituted 3 directly into $4 t^{3}$ to obtain 108 miles $\left(\mathrm{F}_{6}\right)$. This example shows how multidimensional connectivity can be used to find hidden information.

\section{Backcloth, traffic and q-transmission}

Complex systems science requires qualitative (relational) and quantitative (numerical) information to be combined. Let $F$ be a simplicial family and let $f: \sigma \rightarrow \mathbb{R}$ where $\mathbb{R}$ is a number system such as the reals. For example, let $\sigma$ be a choir with vertices singers, and let $f(\sigma)$ be the time they take to sing a song. Then, as R. H. Atkin suggested, $F$ acts as a relatively static backcloth supporting the relatively dynamic traffic of the system $[1,2]$. Other examples include a backcloth of company simplices supporting a traffic of sales and payments.

The concept of $q$-transmission is based on the idea that $q$-connectedness constrains the dynamics of system behaviour, where connected simplices can "infect" each other with changes in their traffic. For example, let the simplices in Fig. 11 be committees with vertices committee members. Four people are members of both $\sigma_{1}$ and $\sigma_{2}$. When committee $\sigma_{2}$ meets these four bring their memory of the business of committee $\sigma_{1}$. Since there are four people their individual (vertex) memories will be reinforced within the group (tetrahedron), e.g. one person may make mention something which the others can reinforce and add to. Thus committee $\sigma_{2}$ is 'infected' with information from committee $\sigma_{1}$. Similarly, committee $\sigma_{3}$ can be infected with information from committee $\sigma_{1}$, even though $\sigma_{1}$ and $\sigma_{3}$ share no vertices. Committee $\sigma_{6}$ only shares one member with committee $\sigma_{3}$ and the transmission of information will be weaker with, potentially, less information being transmitted and the veracity of that information being more questionable. In contrast, committee $\sigma_{4}$ shares a tetrahedral face with $\sigma_{3}$ supporting the transmission of more and higher quality information. Thus, although committee $\sigma_{5}$ is "more distant" from committee $\sigma_{1}$ than $\sigma_{6}$ the quantity and quality of the information it receives from $\sigma_{1}$ may be superior. 


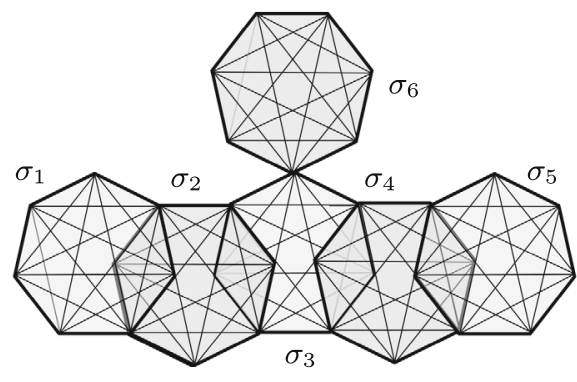

(a) 3-connected 6-dimensional simplices

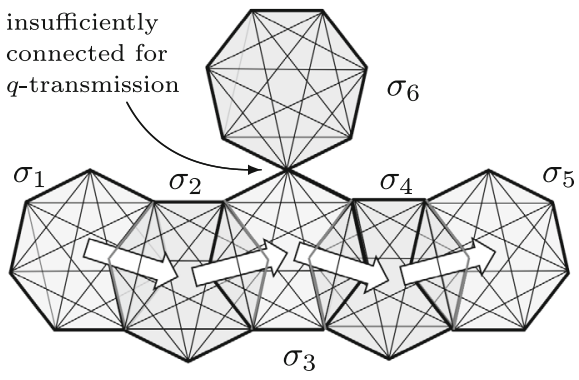

(b) q-transmission dynamics

Fig. 11. Information transmission through $q$-connected simplices.

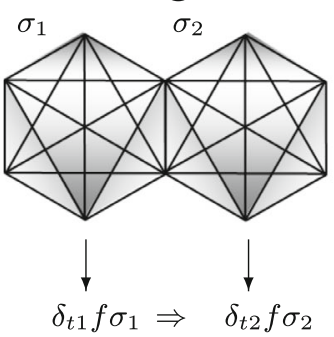

(a) 1-transmission from $\sigma_{1}$ to $\sigma_{2}$

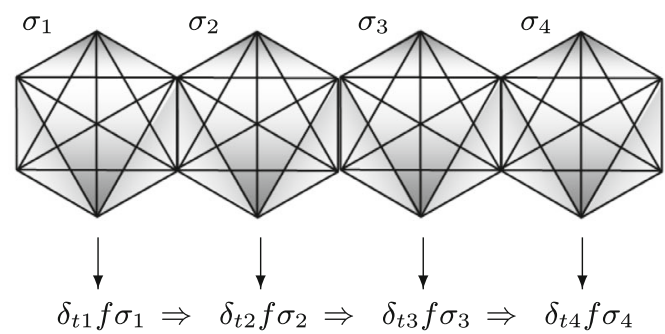

(b) 1-transmission along a chain of 1-connection

Fig. 12. $q$-transmission through shared 1-dimensional faces (edges with 2 vertices).

In Fig. 12(a) $\sigma_{1}$ and $\sigma_{2}$ are two $q$-near simplices in a simplicial family supporting a traffic mapping $f$. Suppose that a change $\delta f \sigma_{1}$ of the value of $f$ on $\sigma_{1}$ causes a change $\delta f \sigma_{2}$ of the value of $f$ on $\sigma_{2}$. Then the change $\delta f$ is said to be $q$-transmitted from $\sigma_{1}$ to $\sigma_{2}$. More generally, change can be $q$-transmitted along chains of $q$-connection, e.g. 1-transmission from $\sigma_{1}$ through $\sigma_{2}$ and $\sigma_{3}$ to $\sigma_{4}$ as illustrated in (Fig. 12(b)).

\section{$q$-transmission fronts}

The general idea behind $q$-transmission is that change will be propagated through a simplicial family in a way conditioned by its $q$-connectivity. Figure 13(a) shows a chain of $q$-connection, and Fig. 13(b) shows how such chains can make up $q$-transmission fronts, through which changes are transmitted, as shown in Fig. 13(c).

Let $\sigma$ be a simplex in the simplicial family $F$. Let $\mathrm{F}_{0}=\left\{\sigma^{0}\right\}$. Let $\mathrm{F}_{1}$ be the set of simplices in $F$ that are $q$-near $\sigma^{0}$. In general let $\mathrm{F}_{k+1}$ be the set of simplices which are $q$-near to a simplex in $\mathrm{F}_{k}$ but not $q$-near any simplices in $\mathrm{F}_{j}$ for $j<k$. The first condition, being $q$-near to a simplex in $\mathrm{F}_{k}$ identifies the new $q$-near simplices. Not being a member of $\mathrm{F}_{j}$ for $j<k$, ensures that the simplices do not belong to previous transmission fronts. Thus the transmission fronts are disjoint sets of simplices.

Q-transmission fronts constrain $q$-transmission dynamics. Change is experienced later and may be weaker at more distant fronts.

The idea of $q$-transmission contrasts with percolation in networks. The $q$-transmission property requires that $q$-dimensional simplices are required for change to be transmitted rather than vertices. A $q$-connected component will be said to $q$-percolate when all its simplices are infected by a change in $f$.

\section{Hypersimplices and hypernetworks}

Hypernetworks are characterised by three major ideas: the first is that of "relational simplex" or hypersimplex; the second is that hypersimplices provide an 


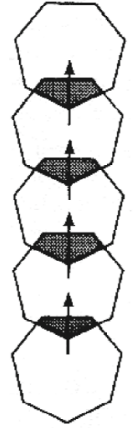

(a) a $q$-chain

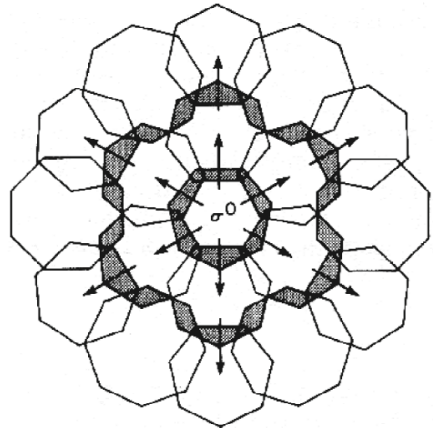

(b) a $q$-transmission front (shaded)

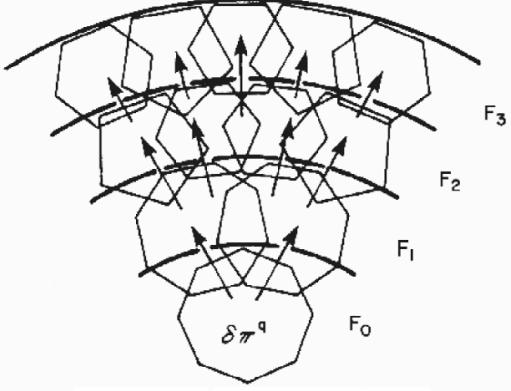

(c) changes propagated through $q$-transmission fronts $\mathrm{F}_{0}, \mathrm{~F}_{1}, \mathrm{~F}_{2}, \mathrm{~F}_{3}$

Fig. 13. $q$-transmission through $q$-transmission fronts.

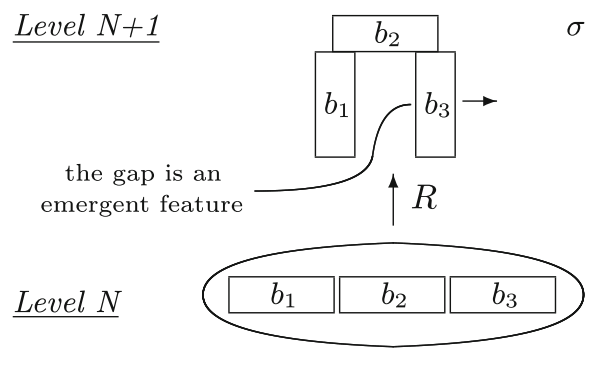

(a) parts assembled into a whole $\sigma=\left\langle b_{1}, b_{2}, b_{3} ; R\right\rangle$

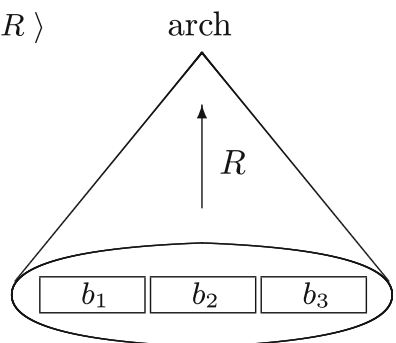

(b) $R$-assembly

(c) a hierarchical cone

Fig. 14. Parts combined into a whole by $R$ forming a relational hypersimplex.

unambiguous way of discriminating levels in multilevel systems; and the third is that these structures can support multilevel system backcloth and traffic dynamics.

A hypersimplex is a simplex that carries its defining relation explicitly following the vertices, e.g. the blocks $b_{1}, b_{2}$ and $b_{3}$ in Fig. 14 are combined by the relation $R$ to create the hypersimplex $\sigma=\left\langle b_{1}, b_{2}, b_{3} ; R\right\rangle$, where the "arch" $\sigma$ exists at a higher more aggregate level than its parts, as shown in Fig. 14.

Hypersimplices provide well defined multilevel aggregation from parts at Level $N$ to structured wholes at Level $N+1$. Fig. 14(b) shows a simplex as a list of parts mapped to a hypersimplex as a structure, $R:\left\langle b_{1}, b_{2}, b_{3}\right\rangle \longrightarrow\left\langle b_{1}, b_{2}, b_{3} ; R\right\rangle$. The partwhole assembly can be represented by hierarchical cones as shown in Fig. 14(c).

Hypernetworks allow many relations on the same vertex set, and generalise multiplex networks [5]. This allows the implementation of multiplex relational algebra:

$$
\left\langle x_{0}, \ldots, x_{p} ; R_{1}\right\rangle \wedge\left\langle x_{0}, \ldots, x_{p} ; R_{2}\right\rangle=\left\langle x_{0}, \ldots, x_{p} ; R_{1} \wedge R_{2}\right\rangle .
$$

\section{Example: The knight fork}

Figure 15 shows three configurations of chess pieces. The configuration on the left, $\left\langle\right.$ rook, knight, king; $\left.R_{1}\right\rangle$, is called a knight fork because the white knight threatens the black rook at the same time that it puts the black king in check. Unless black has a piece that can take it, the white knight can take the more valuable black rook because black must move the king out of check. 


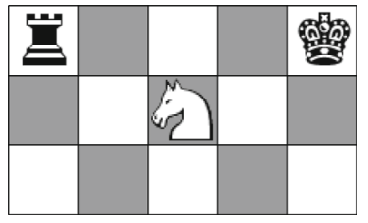

(a) 〈rook, knight, king; $\left.R_{1}\right\rangle$

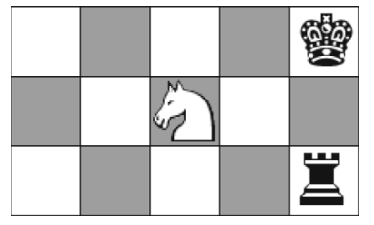

(b) 〈rook, knight, king; $\left.R_{2}\right\rangle$

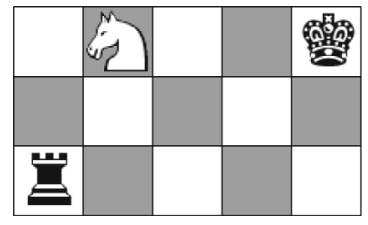

(c) 〈rook, knight, king; $\left.R_{3}\right\rangle$

Fig. 15. Relational structure in chess.<smiles>CCCO</smiles>

(a) n-propyl alcohol<smiles>CC(C)O</smiles>

(b) isopropyl alcohol<smiles>CCOC</smiles>

(c) methyl-ethyl-ether

Fig. 16. Chemical isomers as relational simplices.

The configuration in the centre, 〈rook, knight, king; $\left.R_{2}\right\rangle$, is also a knight fork, but the configuration on the right is not. Thus, the same three pieces are assembled by three different relations, $R_{1}, R_{2}$ and $R_{3}$ to form three different structures.

\section{Example: Chemical Isomers}

Chemical molecules are assemblies of atoms. For example propanol assembles three carbon atoms with eight hydrogen atoms and an oxygen atom, written as $\mathrm{C}_{3} \mathrm{H}_{8} \mathrm{O}$ or $\mathrm{C}_{3} \mathrm{H}_{7} \mathrm{OH}$. Figure 16(a) is $n$-propyl alcohol and Fig. 16(b) is isopropyl alcohol. The oxygen atom is attached to an end carbon atom in the first and to the centre carbon atom in the second, with the $\mathrm{C}-\mathrm{O}-\mathrm{H}$ hydroxyl group substructure common to both. In Fig. 16(c) the oxygen atom is connected to two carbon atoms and there is no C-O-H substructure. This makes it an ether, methyl-ethyl-ether, rather than an alcohol. Thus the relational simplices of the isomers have the same vertices, but the assembly relations are different and the molecules have different emergent properties. The hypersimplex notation discriminates the isomers as follows:

$$
\begin{aligned}
& \left\langle\mathrm{C}, \mathrm{C}, \mathrm{C}, \mathrm{H}, \mathrm{H}, \mathrm{H}, \mathrm{H}, \mathrm{H}, \mathrm{H}, \mathrm{H}, \mathrm{H}, \mathrm{O} ; R_{n-\text { propylalcohol }}\right\rangle \neq \\
& \left\langle\mathrm{C}, \mathrm{C}, \mathrm{C}, \mathrm{H}, \mathrm{H}, \mathrm{H}, \mathrm{H}, \mathrm{H}, \mathrm{H}, \mathrm{H}, \mathrm{H}, \mathrm{O} ; R_{\text {isopropylalcohol }}\right\rangle \neq \\
& \left\langle\mathrm{C}, \mathrm{C}, \mathrm{C}, \mathrm{H}, \mathrm{H}, \mathrm{H}, \mathrm{H}, \mathrm{H}, \mathrm{H}, \mathrm{H}, \mathrm{H}, \mathrm{O} ; R_{\text {methyl-ethyl-ether }}\right\rangle
\end{aligned}
$$

\section{Example: The sun illusion and virtual contours}

Figure $17(\mathrm{a})$ shows the set of lines $\ell_{1}, \ldots, \ell_{16}$ arranged in a circle by the relation $R_{1}$. The resulting structure $\left\langle\ell_{1}, \ldots, \ell_{16} ; R_{1}\right\rangle$ has the emergent property of a central white disk, the so-called sun illusion. Figure $17(\mathrm{~b})$ shows the same set of lines assembled under the relation, $R_{2}$. Now there is no disk but a rectangle shape emerges. Figure 17(c) shows the same sixteen lines assembled by $R_{3}$ so that a virtual contour emerges. Thus the same set of lines can be assembled into three different hypersimplices. 


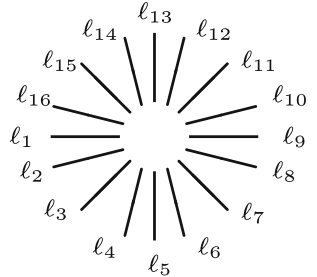

(a) The sun illusion $\sigma_{1}=\left\langle\ell_{1}, \ldots, \ell_{16} ; R_{1}\right\rangle$

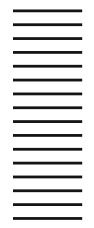

(b) the rectangle illusion $\sigma_{2}=\left\langle\ell_{1}, \ldots, \ell_{16} ; R_{2}\right\rangle$

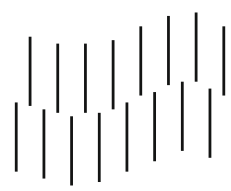

(c) the virtual contour illusion $\sigma_{2}=\left\langle\ell_{1}, \ldots, \ell_{16} ; R_{3}\right\rangle$

Fig. 17. Emergent features in line assemblies.

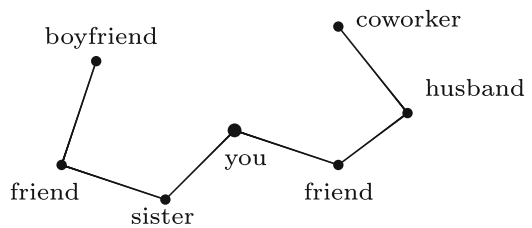

(a) motifs as network structures

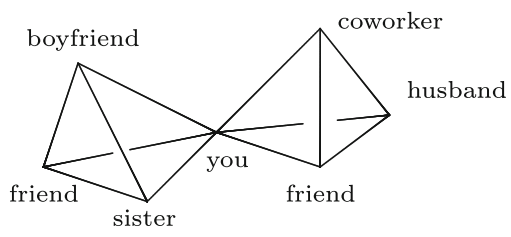

(b) motifs as hypersimplices

Fig. 18. Motifs as hypersimplices.

\section{Example: Motifs and the tetrahedral 3-degree rule}

In their book Connected [6] Christakis and Fowler write "You may not know him personally, but your friend's husband's coworker can make you fat. And your sister's friend's boyfriend can make you thin." However, "Our influence gradually dissipates and ceases to have a noticeable effect on people beyond the social frontier that lies at three degrees of separation." These chains of influence are drawn as paths as shown in Fig. 18. They are examples of motifs, or recurring structural configurations.

Generally motifs are defined by $n$-ary relations, as shown by the vertex removal test, e.g. remove any of $\langle$ you $\rangle$, 〈your friend $\rangle$, 〈your friend's husband $\rangle$, or $\langle$ your friend's husband's co-worker $\rangle$ and the 3-ary relation 'make you fatter' ceases to hold. Thus in this motif the people form a tetrahedral hypersimplex (you, your friend, your friend's husband, your friend's husband's co-worker; $\left.R_{\text {makes_you_fat }}\right\rangle$ as shown in Fig. 18(b).

Christakis and Fowler's "three degree" rule means that these hypersimplices are restricted to tetrahedra. Dunbar gives evidence suggesting that human beings can manage relationships with at most 150-200 other people [7]. Suppose the figure were 100. Then, ignoring repetitions, you are directly influenced by 100 people, each of these is influenced by 100 people, and each of these is influenced by 100 people, so each of us has about a million tetrahedra with the potential to influence us according to the three degree rule. In practice it is less because of the connectivity of people's $q$-transmission fronts, as illustrated in Fig. 13(c).

\section{The multilevel backcloth of complex systems}

Systems may have many levels of representation, e.g. biological systems aggregate from molecules to proteins to cells to organs to organisms. Currently the bottom-up and top-down dynamics of complex systems is poorly understood. Hypersimplices provide a way of representing multilevel aggregation and disaggregation.

Figure 19 shows hierarchical aggregation between three levels, $N+k-1, N+k$ and $N+k+1$. $N$ is an arbitrary but fixed base level. The number $k$ allows the focus to move up and down the levels. 


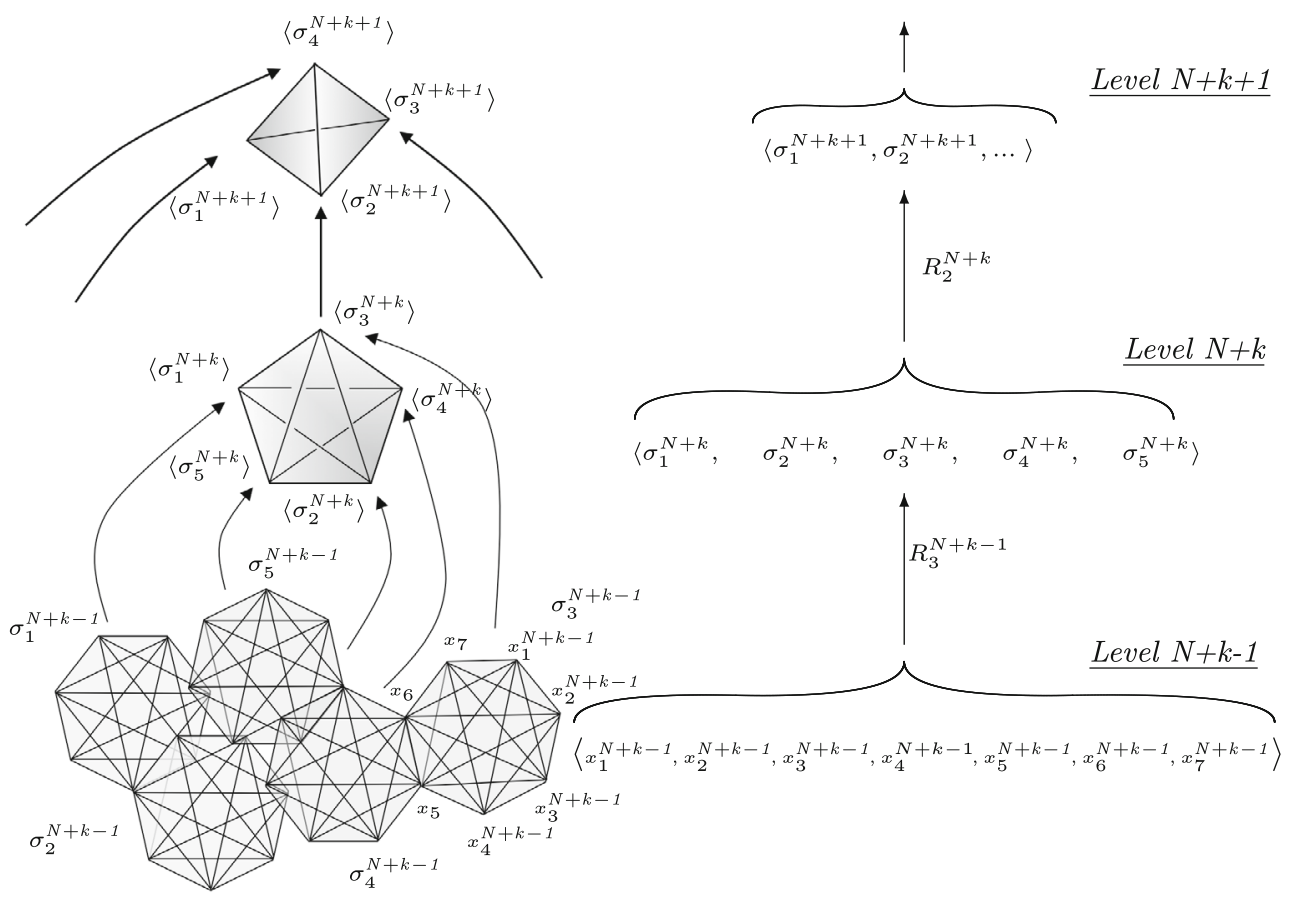

Fig. 19. Multilevel aggregation: Level $N+k$ simplices map to Level $N+k+1$ vertices.

The key idea is that an $n$-ary relation $R_{i}^{N+k}$ maps an ordered set of vertices (simplex) $\left\langle x_{1}^{N+k}, \ldots, x_{n}^{N+k}\right\rangle$ at Level $N+k$ to the hypersimplex $\sigma_{i}^{N+k+1}=\left\langle x_{1}^{N+k}, \ldots, x_{n}^{N+k}\right.$; $\left.R_{i}^{N+k}\right\rangle$ at Level $N+k+1$ where it is treated as a vertex, $\sigma_{i}^{N+k+1} \stackrel{\text { def }}{\equiv} x_{i}^{N+k+1}$.

For example, let vertices $\left\langle x_{1}^{N+k-1}, \ldots, x_{7}^{N+k-1}\right\rangle$ be people employed by a company. They are assembled by the 7 -ary relation $R_{3}^{N+k-1}$ to form the company, $\sigma_{3}^{N+k}$. The company is then treated as a vertex at Level $N+k$. At Level $N+k$ there are five companies assembled by $R_{2}^{N+k}$ into, say, a market $\sigma_{2}^{N+k+1}$ at Level $N+k+1$. Figure 19 shows the following aggregations:

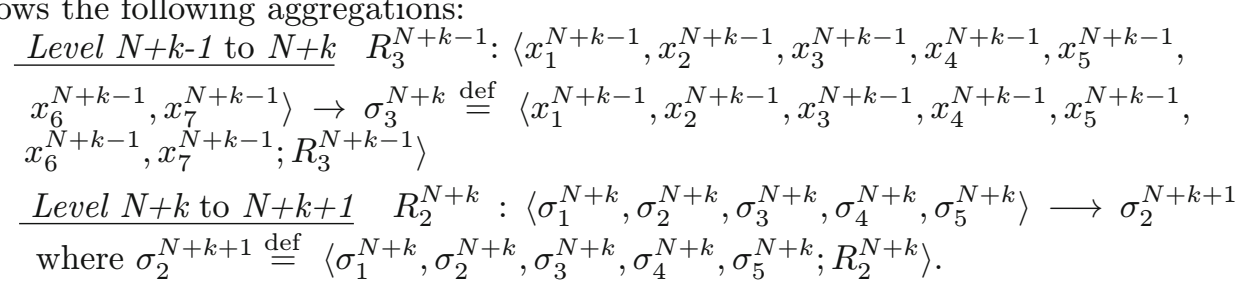

In general the relation $R_{i}^{N+k}$ maps a simplex (an ordered list of Level $N+k$ vertices) to a hypersimplex, $\sigma_{i}^{N+k+1}$, at Level $N+k+1$ where it is treated as a vertex, $x_{i}^{N+k+1}$.

\section{Hierarchical traffic aggregation}

Unifying the dynamics of complex multilevel systems is a major outstanding scientific problem. An objective of hypernetwork theory is to make hierarchical traffic aggregation and disaggregation well defined between backcloth levels $N+k$ and $N+k+1$, and therefore between all levels in multilevel systems. In Fig. 20 let $R_{i}^{N+k}$ be an 


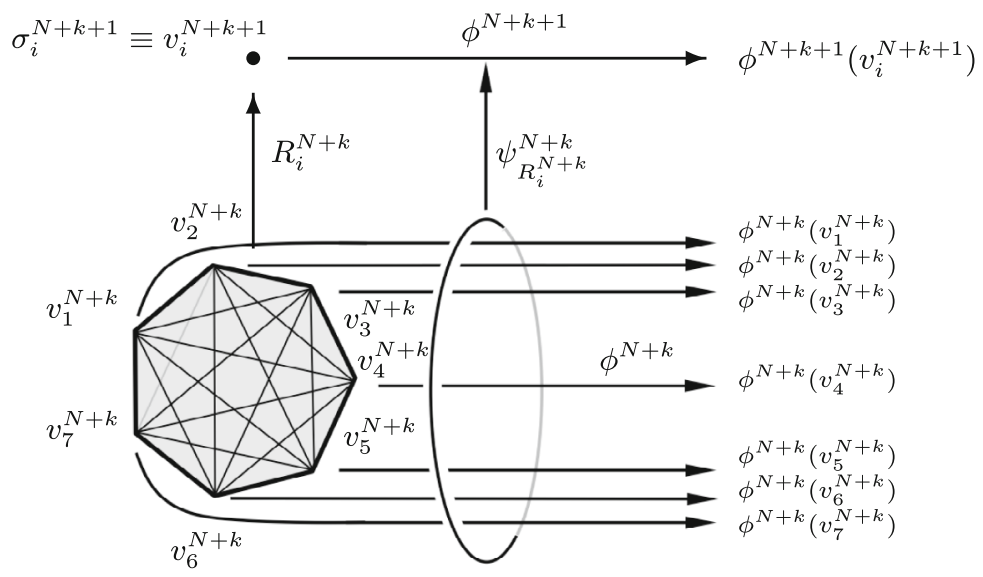

Fig. 20. Multilevel traffic aggregating across the multilevel backcloth.

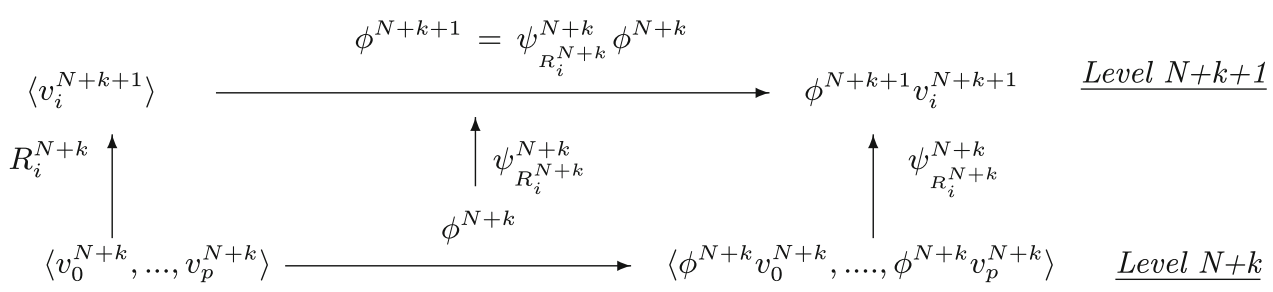

Fig. 21. $\psi_{R_{i}^{N+k}}^{N+k}$ transforms a vertex mapping at Level $N+k$ to a vertex mapping at $N+k+1$.

assembly relation on the simplex $\left\langle v_{1}^{N+k}, \ldots, v_{7}^{N+k}\right\rangle, R_{i}^{N+k}\left\langle v_{1}^{N+k}, \ldots, v_{7}^{N+k}\right\rangle \stackrel{\text { def }}{=}$ $\sigma_{i}^{N+k+1}=\left\langle v_{1}^{N+k}, \ldots, v_{p}^{N+k} ; R_{i}^{N+k}\right\rangle$. This is to be treated as a vertex $v_{i}^{N+k+1}$ at Level $N+k+1$

Let $\phi^{N+k}$ be a mapping of the vertices of the simplex $\left\langle v_{1}^{N+k}, \ldots, v_{p}^{N+k}\right\rangle$ to a number system such as the reals, $\mathbb{R}$. Let

$$
\phi^{N+k}\left\langle v_{1}^{N+k}, \ldots, v_{p}^{N+k}\right\rangle \stackrel{\text { def }}{=}\left\langle\phi^{N+k}\left(v_{1}^{N+k}\right), \ldots, \phi^{N+k}\left(v_{p}^{N+k}\right)\right\rangle
$$

Let $\psi_{R_{i}^{N+k}}^{N+k}:\left\langle\phi^{N+k} v_{1}^{N+k}, \ldots, \phi^{N+k} v_{p}^{N+k}\right\rangle \rightarrow \mathbb{R}$ be a mapping associated with $R_{i}^{N+k}$, Then $\psi_{R_{i}^{N+k}}^{N+k}$ is defined to be a hierarchical traffic aggregation mapping

$$
\psi_{R_{i}^{N+k}}^{N+k}: \phi^{N+k} \rightarrow \phi^{N+k+1}
$$

if the diagram in Fig. 21 commutes with $\phi^{N+k+1} R_{i}^{N+k}=\psi_{R_{i}^{N+k}}^{N+k} \phi^{N+k}$.

Science involves the reconstruction of systems from data. Given a traffic mapping $\phi^{N+k}$ relevant to the dynamics of a system at Level $N+k$ there are two possibilities:

- The mapping $\phi^{N+k}$ can be reconstructed from data at Level $N+k$ or above. In this case the system is said to be information complete at Level $N+k$.

- The mapping $\phi^{N+k}$ cannot be reconstructed from data at Level $N+k$ or above. It is information incomplete. In this case to reconstruct a system's dynamics it is necessary to use lower level data given by functions $\phi^{N+k-i}, i \geq 1$, and to find hierarchical traffic aggregation mappings $\psi_{R_{j}^{N+k-i}}^{N+k-i}$.

Hypernetwork structures, or equivalents, are necessary to address the second case [9]. 


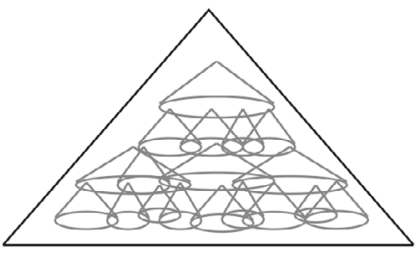

(a) a multilevel triangle

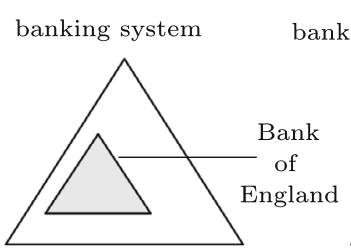

(b) subsystem

regulation system

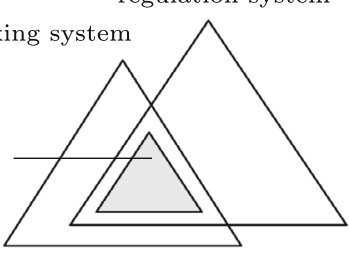

(c) intersection

Fig. 22. Multilevel operator on multilevel triangles.

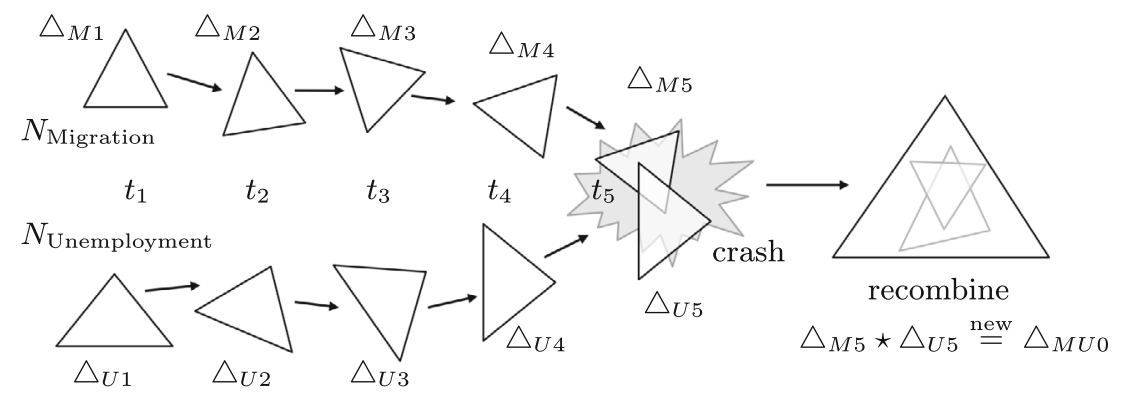

Fig. 23. Multilevel fragment-recombine operators.

\section{The multilevel fragment-recombine operator}

When dealing with multilevel systems it would be useful to have a single symbol to represent the very complicated multilevel cone structures illustrated in Fig. 22(a). One possibility is to enclose them by triangle. This representation allows a subsystem to be represented by a triangle within a triangle as shown in Fig. 22(b). Since the intersection of two triangles is also a triangle, this representation is convenient to denote the intersection of two multilevel systems, as shown in Fig. 22(c).

This representation suggests an exciting new possibility for multilevel complex systems. To be more concrete consider a narrative as a multilevel structure made of words, phrases, paragraphs and complete stories. Narratives are very important in policy and very important for the development of a theory of complex social systems.

For example, Europe is grappling with many narratives associated with migrants, and these narratives work at the level of the plight of individual people, through to more aggregate structures such as people traffickers' boats to more aggregate structures such as countries and their policies. The narratives include political and economic aspects at many level of aggregation. Let this multilevel narrative be called $N_{\text {Migration }}$ as shown top-left in Fig. 23. $\triangle_{M i}$ is the state of the narrative at time $t_{i}$.

Alongside the strong migration UK narrative there are others, e.g. the unemployment narrative, $N_{\text {Unemployment }}$, shown bottom-left in Fig. 23.

Both these narratives evolve in time, with information and invention being added or lost as the meanings of the narrative evolve. Figure 23 shows these narratives evolving independently until they crash into each other at time $t_{5}$. The combinatorial dynamics of such a crash is not well understood, but it involves parts of the two multilevel systems interacting and each of the multilevel narratives fragmenting before they recombine to form new composite narratives, e.g. $N_{\text {migrants_are_taking_our_jobs }}$.

Let the fragment-recombine operator of multilevel systems, $\star$, be defined as

$$
\star:\left(\Delta_{1}, \Delta_{2}\right) \rightarrow \Delta_{1} \star \Delta_{2}
$$


where $\Delta_{1}$ and $\Delta_{2}$ are multilevel systems before they crash and $\Delta_{1} \star \Delta_{2}$ is the multilevel system after. The multilevel $\star$ operator is completely new and developing its properties is a great opportunity for mathematicians, informaticians and social scientists.

\section{Conclusions: Research challenges in hypernetwork theory}

Hypernetworks have been presented as a generalisation of hypergraph theory, network theory, and multiplex network theory. There remain many research challenges in hypernetwork theory including:

- understanding better the multilevel interactions of backcloth and traffic dynamics, including $q$-transmission, chain length, and information completeness.

- hypernetworks allow many relations between vertices. The formulation of hypersimplices allows new algebraic operations to be defined between $n$-ary relations, e.g. $\left\langle x_{0}, \ldots, x_{p} ; R_{1}\right\rangle \diamond\left\langle x_{0}, \ldots, x_{p} ; R_{2}\right\rangle=\left\langle x_{0}, \ldots, x_{p} ; R_{1} \diamond R_{2}\right\rangle$

- the nature of hypersimplex intersection, $\left\langle x_{1}, x_{2}, \ldots, x_{n} ; R_{1}\right\rangle \cap\left\langle y_{1}, y_{2}, \ldots, y_{p^{\prime}} ; R_{2}\right\rangle$ $\stackrel{?}{=}\left\langle z_{1}, z_{2}, \ldots, z_{q} ; R_{1} \wedge R_{2}\right\rangle$, where $\left\{z_{1}, z_{2}, \ldots, z_{q}\right\}=\left\{x_{1}, x_{2}, \ldots, x_{p}\right\} \cap\left\{y_{1}, y+\right.$ $\left.2, \ldots, y_{p^{\prime}}\right\}$.

- the multilevel fragment-recombine operator $\star:\left(\triangle_{1}, \triangle_{2}, \ldots\right) \rightarrow \triangle_{1}, \star \triangle_{2} \star \ldots$ for multilevel systems presents many new opportunities and challenges.

\section{References}

1. R.H. Atkin, I.J. Man-Machine Studies 4, 139 (1972)

2. R.H. Atkin Combinatorial connectivities in social systems, Birkhäuser (Basel) (1977)

3. C. Berge, 'Sur certains hypergraphes généralisant les graphes bipartites'. In P. Erdös, A. Rhényi, V.T. Sós (eds), Combinatorial Theory and its Applications I, (Proc. Colloquium on Combinatorial Theory and its Applications), 119. North-Holland, 1970

4. C. Berge, Hypergraphs: Combinatorics of Finite Sets (North Holland Amsterdam 1989)

5. S. Boccalettia, et al., Phys. Rep. 544, 1 (2014), https://arxiv.org/abs/1407.0742

6. N. Christakis, J. Fowler, Connected: The amazing power of social networks and how they shape our lives, (Harpur Press, London 2010)

7. R.I.M. Dunbar, J Hum. Evolu. 22, 469 (1992)

8. L.C. Freeman, D.R. White, 1993. 'Using Galois lattices to represent network data', Sociological methodology, Volume 23, American Sociological Association, ISBN 1-55786464-0, ISSN 0081-1750, http://eclectic.ss.uci.edu/ drwhite/pw/Galois.pdf

9. J.H. Johnson, Hypernetworks in the science of complex systems, (Imperial College Press London 2014), http://www.hypernetworks.eu

Open Access This is an Open Access article distributed under the terms of the Creative Commons Attribution License (http://creativecommons.org/licenses/by/4.0), which permits unrestricted use, distribution, and reproduction in any medium, provided the original work is properly cited. 